August, 1994.

hep-ph/9408367

McGill-94/37, NEIP-94-007, UMD-PP-95-11

\title{
Heavy Sterile Neutrinos
}

\author{
and \\ Neutrinoless Double Beta Decay \\ P. Bamert ${ }^{a}$, C.P. Burgess ${ }^{a, b}$ and R.N. Mohapatra ${ }^{c}$ \\ ${ }^{a}$ Institut de Physique, Université de Neuchâtel \\ 1 Rue A.L. Breguet, CH-2000 Neuchâtel, Switzerland. \\ ${ }^{b}$ Physics Department, McGill University \\ 3600 University St., Montréal, Québec, Canada, H3A 2 T8. \\ ${ }^{c}$ Department of Physics, University of Maryland \\ College Park, Maryland, USA, 20742.
}

\begin{abstract}
We investigate the possibility of producing neutrinoless double beta decay without having an electron neutrino with a mass in the vicinity of $1 \mathrm{eV}$. We do so by having a much lighter electron neutrino mix with a much heavier $(m \gtrsim 1 \mathrm{GeV})$ sterile neutrino. We study the constraints on the masses and mixings of such heavy sterile neutrinos from existing laboratory, astrophysical and cosmological information, and discuss the properties it would require in order to produce a detectable signal in current searches for neutrinoless double beta decay.
\end{abstract}




\section{Introduction}

Our ultimate understanding of the universe relies on two crucial ingredients: the nature of the constituents (or building blocks), and the nature of the forces through which they interact. Experience to date indicates that spin-half fermions are the ultimate constituents, whereas forces arise from the exchange of gauge bosons associated with local symmetries, and Higgs bosons needed to break these symmetries. The highly successful Standard Model (SM) is based on 45 chiral fermions (15 for each generation consisting of twelve coloured quark states and three leptonic ones). Of these, the neutrinos have the unique property of being electrically neutral.

A necessary part of the exploration of physics beyond the SM is the study of new chiral fermions and their properties. Apart from sheer curiosity regarding their existence, there are often very good physical motivations for postulating such particles. For instance, it is by now well known that if neutrinos have a mass, the smallness of this mass is easily understood if there is an additional heavy, isosinglet Majorana neutrino which mixes with the known ones via the so-called see-saw mechanism [1]. Similarly, there also exist quite good reasons which would motivate the existence of new charged fermions not present in the standard model. An important area of investigation in particle physics now is the study of the constraints that may be inferred on the properties of such new fermions using the existing data, as well as the identification of new experiments which can further constrain these properties.

Since charged fermions cannot be singlets under the SM gauge group, the LEP data on the $Z$-width already imposes very stringent limits on their masses, i.e. $m_{F} \gtrsim 45 \mathrm{GeV}$. However, no such direct constraints need apply for SM-singlet particles, unless they mix strongly with the known neutrinos. There are, however, many indirect limits on such particles [2], [3], [4], [5], [6], [7] and our goal in this paper is to explore these in the context of a specific model and indicate how future neutrinoless double beta decay $\left(\beta \beta_{0 \nu}\right)$ experiments can probe the existence of these particles in interesting ranges of masses and mixing angles.

One motivation for examining the particular type of models we consider here is that they contradict the maxim ${ }^{1}$ that the observation of neutrinoless double-beta decay would demonstrate the existence of a mass for the electron neutrino in the range of $1 \mathrm{eV}$ [10]. In

1 Alternative scenarios have also been explored for having observable $\beta \beta_{0 \nu}$ decay without requiring 
the models we study, all of the neutrinos are either much lighter than, or much heavier than the $\mathrm{eV}$ mass region. Neutrinoless double beta decay proceeds in these models through the virtual exchange of the heavier (i.e. GeV-scale or higher) neutrinos. We are able to find phenomenologically acceptable masses and mixings for such a model that are consistent with an observable signal for double beta decay.

\section{The Model}

We restrict ourselves for simplicity to supplementing the SM with two sterile neutrinos, $N_{ \pm}$, which mix only with the SM neutrinos of the first generation. The reasons for adding two sterile neutrinos rather than one are twofold: (a) the case of one extra sterile neutrino falls into a subclass of models which use the see-saw mechanism, and which have been discussed elsewhere [3], [4]; and (b) in models with one sterile neutrino, in the limit $m_{\nu_{e}} \rightarrow$ 0 , the sterile neutrino completely decouples and becomes invisible, whereas in models with two (or more) sterile neutrinos, the situation completely changes, and the sterile neutrinos can mix appreciably even in the limit $m_{\nu_{e}} \rightarrow 0$, and so can be potentially visible in many processes [5].

The interaction lagrangian involving $N_{ \pm}$can be written as follows:

$$
\mathcal{L}=\mathcal{L}_{0}+\mathcal{L}_{1}
$$

where $\mathcal{L}_{0}$ conserves lepton number and $\mathcal{L}_{1}$ violates it. (We assign $L\left(N_{ \pm}\right)= \pm 1$ to the left-handed parts of $N_{ \pm}$.) The most general renormalizable couplings and masses are:

$$
\mathcal{L}_{0}=-m \bar{N}_{+} \gamma_{L} N_{-}-\lambda_{e}\left(\bar{L}_{e} \gamma_{L} N_{-}\right) H+\text { h.c. }
$$

and

$$
\mathcal{L}_{1}=-\frac{\mu_{+}}{2} \bar{N}_{+} \gamma_{L} N_{+}-\frac{\mu_{-}}{2} \bar{N}_{-} \gamma_{L} N_{-}-\tilde{\lambda}_{e}\left(\bar{L}_{e} \gamma_{L} N_{+}\right) H+\text { h.c. }
$$

where $H$ is the usual SM Higgs doublet, and $L_{e}=\left(\begin{array}{c}\nu_{e} \\ e\end{array}\right)$ is the first-generation lepton doublet. After the SM gauge symmetry breaking — with $\langle H\rangle=v=174 \mathrm{GeV}$ - one gets

\footnotetext{
$m_{\nu_{e}}$ to be in the eV range [8]. Our approach here is more similar to, and updates, the framework of Ref. [9].
} 
the following most general mass term relating the left-handed states $\nu_{e}, N_{+}$and $N_{-}$:

$$
\mathcal{L}_{m}=-\frac{1}{2}\left(\begin{array}{c}
\bar{\nu}_{e} \\
\bar{N}_{+} \\
\bar{N}_{-}
\end{array}\right)^{T}\left(\begin{array}{ccc}
0 & \tilde{\lambda}_{e} v & \lambda_{e} v \\
\tilde{\lambda}_{e} v & \mu_{+} & m \\
\lambda_{e} v & m & \mu_{-}
\end{array}\right)\left(\begin{array}{c}
\nu_{e} \\
N_{+} \\
N_{-}
\end{array}\right) .
$$

We assume for simplicity, in writing eq. (4), that the elements of the mass matrix are real. This mass matrix has three nonzero eigenvalues, one of which is the physical electron neutrino, $\nu_{e}^{\prime}$. Since the direct laboratory bounds on the mass of a dominantly electron neutrino are quite low, we must ask under what circumstances a massless neutrino can emerge from this matrix. There are two possible regimes:

(1) $\tilde{\lambda}_{e}=\mu_{+}=0$. The mass eigenstates can in this case be written as:

$$
\left(\begin{array}{c}
\nu_{e}^{\prime} \\
N_{+}^{\prime} \\
N_{-}^{\prime}
\end{array}\right)=\left(\begin{array}{ccc}
c_{1} & -s_{1} & 0 \\
s_{1} c_{2} & c_{1} c_{2} & -s_{2} \\
i s_{1} s_{2} & i c_{1} s_{2} & i c_{2}
\end{array}\right)\left(\begin{array}{c}
\nu_{e} \\
N_{+} \\
N_{-}
\end{array}\right)
$$

where $c_{i}\left(s_{i}\right)$ denotes $\cos \theta_{i}\left(\sin \theta_{i}\right)$ while

$$
\tan \theta_{1}=\frac{\lambda_{e} v}{m} ; \quad \text { and } \quad \tan 2 \theta_{2}=2 \frac{\sqrt{m^{2}+\lambda_{e}{ }^{2} v^{2}}}{\mu_{-}} .
$$

Finally, the factors of ' $i$ ' in the last row of eq. (5) come from the chiral rotation that is required to ensure that all of the entries in the final mass matrix are positive. The corresponding masses are:

$$
M_{\nu_{e}^{\prime}}=0, \quad \text { and } \quad M_{N_{ \pm}^{\prime}}=\frac{1}{2}\left[\sqrt{\mu_{-}^{2}+4\left(m^{2}+\lambda_{e}^{2} v^{2}\right)} \mp \mu_{-}\right]
$$

Notice that in the limit $\mu_{-} \rightarrow 0$, lepton number is exactly conserved, and $N_{ \pm}^{\prime}$ forms a degenerate Dirac pair. In this case all lepton-number violating processes, such as $\beta \beta_{0 \nu}$ decay, are forbidden.

(2) $\lambda_{e}=\mu_{-}=0$. This alternative is identical to case (1) above, but with the two sterile states $N_{ \pm}$interchanged. The physical implications of this case are therefore identical to case (1), and we need not further pursue this alternative separately. We henceforth exclusively focus on mass-matrix parameters that are in the vicinity of case (1). 
For later purposes it is useful to define a dimensionless parameter, $\epsilon$, which measures the strength of the lepton-number violation in the mass matrix:

$$
\epsilon=\frac{\mu_{-}}{\sqrt{m^{2}+\lambda_{e}^{2} v^{2}}}
$$

The ratio of the nonzero mass eigenvalues have a simple expression in terms of this parameter:

$$
\frac{M_{N_{-}^{\prime}}}{M_{N_{+}^{\prime}}}=\frac{\sqrt{1+4 / \epsilon^{2}}+1}{\sqrt{1+4 / \epsilon^{2}}-1}=\cot ^{2} \theta_{2} .
$$

In the lepton-conserving limit, $\epsilon \ll 1, M_{N_{-}^{\prime}} \simeq M_{N_{+}^{\prime}}$ as would be expected for pseudo-Dirac particles. In this case the mass eigenstates, $N_{ \pm}^{\prime}$, have opposite CP properties. For $\epsilon \gg 1$ we instead have $M_{N_{-}} / M_{N_{+}} \sim \epsilon^{2}$, so that in this limit $N_{-}^{\prime}$ is much heavier than $N_{+}^{\prime}$.

We could now turn on small non-vanishing values for $\tilde{\lambda}_{e}$ and $\mu_{+}$, so that the electron neutrino acquires a small nonzero mass. However, we are interested in the situation for which $M_{\nu_{e}} \ll 1 \mathrm{eV}$ and yet for which there are nevertheless potentially observable contributions to $\beta \beta_{0 \nu}$ decay. We therefore keep $\tilde{\lambda}_{e}$ and $\mu_{+}$negligibly small in what follows, although we return to the naturalness of this choice in the following section. We envision the possibility that the $N_{ \pm}^{\prime}$ masses span a wide range of possible values, starting from the $\mathrm{MeV}$ up to the $\mathrm{GeV}$ range. Clearly, the properties of such particles are highly constrained by known laboratory, astrophysical and cosmological information. We discuss the ranges of masses and mixings that are not already ruled out by these constraints and see if $\beta \beta_{0 \nu}$ decay can be observable in the allowed range.

\section{Contributions to $\beta \beta_{0 \nu}$ Decay}

As may be seen from eq. (5), the heavy sterile neutrinos acquire charged-current weak interactions through their mixing with the electroweak eigenstate $\nu_{e}$, resulting in Kobayashi-Maskawa-type mixing angles

$$
U_{e \nu_{e}^{\prime}}=c_{1}, \quad U_{e N_{+}^{\prime}}=s_{1} c_{2}, \quad U_{e N_{-}^{\prime}}=-i s_{1} s_{2} .
$$

Provided that $\mu_{-} \neq 0$, so that lepton number is broken, neutrinoless double beta decay arises in this model via the exchange of the three neutrino states. The differential decay 
rate for this decay between two $0^{+}$nuclei can be written in the following simple form [11]:

$$
\frac{d \Gamma}{d \varepsilon_{1} d \varepsilon_{2}}=\frac{G_{F}^{4} \cos ^{4} \theta_{c}}{2 \pi^{3}}|\mathcal{W}|^{2} \delta\left(Q-\varepsilon_{1}-\varepsilon_{2}\right)\left[\varepsilon_{1} p_{1} F\left(\varepsilon_{1}\right)\right]\left[\varepsilon_{2} p_{2} F\left(\varepsilon_{2}\right)\right]
$$

where $G_{F}$ is Fermi's constant; $\theta_{c}$ is the Cabbibo angle which governs the strength of the hadronic charged-current; $\varepsilon_{i}$ and $p_{i}$ are the energy and momentum of each of the final two electrons; $Q=M(Z, A)-M(Z+2, A)-2 m_{e}$ is their endpoint energy - typically several $\mathrm{MeV}$; and $F(\varepsilon)$ is the Fermi function which describes the distortion of the electron spectrum due to the nuclear charge. $Z$ and $A$ represent the charge and mass number of the initial nucleus. For our present purposes it is convenient to work with analytic expressions for the total decay rate, which we can obtain if we make some simplifying assumptions, which are sufficiently accurate for the estimates in this paper. In performing the phasespace integrals we therefore neglect: $(i)$ the electron mass, and $(i i)$ the Coulomb-distortion factor, $F(\varepsilon)$.

The dependence on the neutrino masses, in eq. (11), lies in the quantity $\mathcal{W}$, which is given explicitly by:

$$
\mathcal{W}=\sum_{i} U_{e i}^{2} m_{i} \int \frac{d^{4} p}{(2 \pi)^{4}}\left(\frac{w}{p^{2}-m_{i}^{2}+i \varepsilon}\right) .
$$

The sum here is over all three neutrino species. $w=w\left(p_{0},|\mathbf{p}|\right)$ represents a particular Lorentz-invariant combination of form factors describing the nuclear matrix element of the two hadronic charged currents. All of the theoretical uncertainty in the decay rate enters with the estimating of $w$ within a model of the nucleus. The connection between $\mathcal{W}$ as defined here and the usual estimates [12], [13], [14], based on an independent-nucleon model of the nucleus within the closure approximation, is given by [11]:

$$
\mathcal{W}=\sum_{i} \frac{U_{e i}^{2} m_{i}}{4 \pi}\left\langle N^{\prime}(Z+2, A)\left|\sum_{m n} \tau_{m}^{+} \tau_{n}^{+} h\left(\mathbf{r}_{n m} ; m_{i}\right)\left(g_{V}^{2}-g_{A}^{2} \vec{\sigma}_{n} \cdot \vec{\sigma}_{m}\right)\right| N(Z, A)\right\rangle
$$

where $m$ and $n$ run over the labels of the nucleons in the nucleus; $\tau_{m}^{+}$and $\vec{\sigma}_{m}$ are isospin and spin matrices for the $m$ th nucleon; and $g_{V}$ and $g_{A}$ are the vector and axial chargedcurrent couplings of the nucleon. The function $h\left(\mathbf{r}_{m n} ; m_{i}\right)$ of the internucleon separation, $\mathbf{r}_{m n}$, is the neutrino potential, which is defined by the following integral:

$$
h(\mathbf{r} ; m)=\frac{1}{2 \pi^{2}} \int d^{3} \mathbf{p} \frac{\exp (-i \mathbf{p} \cdot \mathbf{r})}{\omega(\omega+\mu)} ; \quad \omega=\sqrt{\mathbf{p}^{2}+m^{2}},
$$


where $\mu=\frac{1}{2}[M(Z, A)+M(Z+2, A)]$ is the mean excitation energy of the nucleus.

For the present purposes, however, we need not use the detailed matrix-element machinery, as quite good analytic results can be obtained by making the following simplifying approximation for the functional form for $w$. We parameterize $w$ by representing it as a step functions in energy and momentum: $w\left(p_{0},|\mathbf{p}|\right) \simeq w_{0} \Theta\left(p_{0}-E_{F}\right) \Theta\left(|\mathbf{p}|-p_{F}\right)$. Here $p_{F}$ represents the nucleon Fermi momentum and $E_{F}=p_{F}^{2} / 2 m_{N}$ is the corresponding Fermi energy. By requiring this approximation to reproduce the observed $\beta \beta_{2 \nu}$ decay rates, we find $w_{0} \simeq 4 \mathrm{MeV}^{-1}$, and by requiring that the present upper limit on the half life for neutrinoless decay [15] imply an upper limit for light neutrino masses [16] of $\left|U_{e \nu}^{2} m_{\nu}\right| \lesssim 2$ $\mathrm{eV},{ }^{2}$ we find $p_{F} \simeq 60 \mathrm{MeV}$ (and so $E_{F} \simeq 2 \mathrm{MeV}$ ).

With these choices we obtain the following expression for the total $\beta \beta_{0 \nu}$ decay rate:

$$
\Gamma\left(\beta \beta_{0 \nu}\right) \simeq \frac{G_{F}^{4} Q^{5} \cos ^{4} \theta_{c}}{60 \pi^{3}}|\mathcal{W}|^{2}
$$

with $\mathcal{W}$ given by an elementary integral:

$$
\begin{aligned}
\mathcal{W} & \simeq \frac{i w_{0}}{4 \pi^{3}} \sum_{i} U_{e i}^{2} m_{i} \int_{0}^{E_{F}} d u\left[p_{F}-\sqrt{u^{2}+m_{i}^{2}} \tan ^{-1}\left(\frac{p_{F}}{\sqrt{u^{2}+m_{i}^{2}}}\right)\right], \\
& \approx \frac{i w_{0}}{4 \pi^{3}} \sum_{i} U_{e i}^{2} m_{i}\left\{E_{F} p_{F}-\frac{\pi E_{F}^{2}}{4}-\frac{\pi m_{i}^{2}}{4}\left[\log \left(\frac{2 E_{F}}{m_{i}}\right)+\frac{1}{2}\right]\right\}+\cdots \quad\left(m_{i} \ll E_{F} \ll p_{F}\right), \\
& \approx \frac{i w_{0}}{4 \pi^{3}} \sum_{i} \frac{U_{e i}^{2} E_{F} p_{F}^{3}}{3 m_{i}}+\cdots \quad\left(E_{F} \ll p_{F} \ll m_{i}\right) .
\end{aligned}
$$

Motivated by the expression for the decay rate due to light neutrinos, it has become conventional to quote the experimental limit on $\beta \beta_{0 \nu}$ decay as an upper limit on the 'effective' neutrino mass, defined by $m_{\nu}^{\text {eff }}=\sum_{i} U_{e i}^{2} m_{i}$. As mentioned previously, the current experimental limit [15] implies an upper bound $m_{\nu}^{\text {eff }} \lesssim 2 \mathrm{eV}$ [16]. From the above formulae for $\Gamma\left(\beta \beta_{0 \nu}\right)$ we can obtain similar constraints on $U_{e i}$ and $m_{i}$ for arbitrary neutrino masses. For the model under study here, we assume the $\nu_{e}^{\prime}$ mass to be too small to contribute, and so there are three limiting cases to consider:

2 We take here a bound which is twice as large as the usually-quoted limit, since we allow for an uncertainty in the nuclear matrix elements of a factor of 2. 
(1) If the two sterile neutrinos are both light compared to a few $\mathrm{MeV}$, then one would expect $m_{\nu}^{\text {eff }}=s_{1}^{2}\left(M_{N_{+}^{\prime}} c_{2}^{2}-M_{N_{-}^{\prime}} s_{2}^{2}\right)$ as the approximate expression for $m_{\nu}^{\text {eff }}$. However, in the model considered here this quantity vanishes, as may be seen from eq. (9). As a result we must work to sub-leading order in the sterile neutrino masses when approximating the integral in eq. (16), leading to

$$
m_{\nu}^{\mathrm{eff}}=\frac{\pi s_{1}^{2}}{4 E_{F} p_{F}}\left|M_{N_{+}^{\prime}}^{3} c_{2}^{2}\left[\frac{1}{2}+\log \left(\frac{2 E_{F}}{M_{N_{+}^{\prime}}}\right)\right]-M_{N_{-}^{\prime}}^{3} s_{2}^{2}\left[\frac{1}{2}+\log \left(\frac{2 E_{F}}{M_{N_{-}^{\prime}}}\right)\right]\right| \lesssim 2 \mathrm{eV}
$$

(2) If both sterile neutrinos have large masses compared to $p_{F} \simeq 60 \mathrm{MeV}$, then we instead find

$$
\frac{s_{1}^{2} p_{F}^{2}}{3}\left|\frac{c_{2}^{2}}{M_{N_{+}^{\prime}}}-\frac{s_{2}^{2}}{M_{N_{-}^{\prime}}}\right| \lesssim 2 \mathrm{eV}
$$

(3) Finally, if $M_{N_{+}^{\prime}} \ll p_{F}$ and $M_{N_{-}^{\prime}} \gg p_{F}$, then

$$
s_{1}^{2}\left|M_{N_{+}^{\prime}} c_{2}^{2}-\frac{s_{2}^{2} p_{F}^{2}}{3 M_{N_{-}^{\prime}}}\right| \lesssim 2 \mathrm{eV}
$$

With these estimates we can determine the masses and couplings of the sterile neutrinos which are consistent with the present non-observation of $\beta \beta_{0 \nu}$. The constraints we obtain for $U_{e i}$ and $m_{i}$ in this way are plotted in Figs. (1). The area below the curves in this figure represents the allowed range. To see if a sterile neutrino will make observable contributions to $\beta \beta_{0 \nu}$ decay, we must see if the range of values which lie close to those curves are consistent with other constraints.

\section{1) Radiative Corrections}

Our estimate of the $\beta \beta_{0 \nu}$ decay rate in this section assumes a negligible contribution from the exchange of the very light neutrino mass eigenstate, $\nu_{e}^{\prime}$. This assumption requires some justification in parts of the parameter space which we consider here. We therefore briefly pause to provide this justification.

The neglect of the light neutrino contribution to $\beta \beta_{0 \nu}$ relies on our decision to choose this mass to be much lighter than $1 \mathrm{eV}$. At tree level this can always be accomplished by 
choosing the parameters $\tilde{\lambda}_{e}$ and $\mu_{+}$to be sufficiently small. A naturalness problem can arise, however, if $m_{\nu_{e}^{\prime}}$ is chosen to be too small in comparison with the parameter $\mu_{-}$, which provides the lepton-number violating contribution to the heavy-neutrino masses. This is because a nonzero $\mu_{-}$generates, through loops, nonzero contributions to $\tilde{\lambda}_{e}$ and $\mu_{+}$, and if these loop-induced contributions are large enough, then the $\nu_{e}^{\prime}$ exchange graph can only be neglected in $\beta \beta_{0 \nu}$ if the loop-induced mass is cancelled by the tree-level term. A naturalness problem arises if the required cancellation becomes implausibly precise.

More quantitatively, we imagine our model to be an effective theory which is obtained after some unknown physics above some scale $\Lambda$ has been integrated out. We then use the renormalization group to run the couplings in this effective theory down from the scale $\Lambda$ to the much lower energies that are relevant for $\beta \beta$ decay. In this way we can compute the contribution to the $\nu_{e}^{\prime}$ mass which is produced by the mixing between the parameters $\tilde{\lambda}_{e}$ and $\mu_{+}$, and $\mu_{-}$as they are run down from the scale $\Lambda$. We regard the theory to be natural if these contributions to the $\nu_{e}^{\prime}$ mass are not larger than, say, $O(1 \mathrm{eV})$, and so do not need to be carefully cancelled by a 'bare' contribution to $m_{\nu_{e}^{\prime}}$ at the scale $\Lambda$.

The dominant graph to consider is that of Fig. (2), in which a SM Higgs scalar is emitted and absorbed by the light neutrino state. Its contribution to the light-neutrino mass is, in order of magnitude:

$$
\delta M_{\nu_{e}^{\prime}}(\mu) \sim\left(\frac{\lambda_{e}}{4 \pi}\right)^{2} \mu_{-} \log \left(\frac{\Lambda^{2}}{\mu^{2}}\right)
$$

For numerical purposes we take the logarithm in this expression to be unity. Requiring the rest of the result to be smaller than $O(1 \mathrm{eV})$ then produces the bound labelled $N N$ in Fig. (3).

\section{Phenomenological Constraints}

We now turn to the exploration of the other constraints on this model. We consider in turn the limits coming from $(i)$ supernova SN1987a, (ii) nucleosynthesis, and (iii) laboratory limits. 


\section{1) Supernova SN1987a}

The first constraint we consider is for low-mass sterile leptons ('low-mass' here means masses smaller than $\sim 50 \mathrm{MeV}$ ) that mix significantly with $\nu_{e}$. Any such particle can be produced in the core of a supernova, where temperatures are typically of order $T_{S N} \simeq$ $(60-70) \mathrm{MeV}$. This must be forbidden since otherwise the supernova would cool too much to be in agreement with the observations of SN1987a. The analysis we require is very similar to the case of right-handed neutrinos discussed in Ref. [17], where it was shown that these considerations lead to the following bounds

$$
3 \times 10^{-2} \lesssim\left|U_{e i}\right|, \text { or else }\left|U_{e i}\right| \lesssim 10^{-5} .
$$

The lower bound comes because for sufficiently strong mixing, the produced sterile neutrinos get trapped in the supernova and so they do not provide a mechanism for cooling too quickly. The upper bound comes from the requirement that not too many sterile neutrinos be radiated by the ordinary particles in the supernova. These bounds are displayed in Fig. (3) by the vertical and horizontal lines labelled $S N$. The vertical line is due to this bound being independent of the neutrino mass, provided only that this mass is much smaller than $T_{S N}$. For the models of interest here, the upper bounds we obtain in this way for $M_{i}$ and $\left|U_{e i}\right|$ imply that the effective mass, $m_{\nu}^{\text {eff }}$, which appears in $\beta \beta_{0 \nu}$ decay can be at most $m_{\nu}^{\text {eff }} \lesssim 5 \times 10^{-3} \mathrm{eV}$. This is well beyond the reach of present- and next-generation [18] $\beta \beta$-decay experiments. It is, however, worth noting that for $M_{N_{ \pm}^{\prime}} \gtrsim 50 \mathrm{MeV}$, this bound is ineffective, and so SN1987a cannot rule out a significant contribution of sterile neutrinos to the $\beta \beta_{0 \nu}$ decay rate, provided that these neutrinos are in this larger-mass regime.

We therefore now turn to the constraints on $N_{ \pm}^{\prime}$ which apply if the masses are $50 \mathrm{MeV}$ or higher.

\section{2) Cosmological Constraints}

One of the major triumphs of the standard hot big-bang model of cosmology is its ability to naturally explain the primordial abundance of the light elements $-{ }^{4} \mathrm{He},{ }^{7} \mathrm{Li}$, ${ }^{2} \mathrm{H}$ and ${ }^{3} \mathrm{He}$ - using the standard model (SM) of electroweak interactions [19]. This, in 
turn, implies stringent bounds on any new physics beyond the SM that involves weaklyinteracting particles. In particular, in the model of present interest, the sterile neutrinos can upset the success of the nucleosynthesis discussion unless their masses and mixings are suitably constrained.

The basic condition is to ensure that the energy density at the nucleosynthesis temperature, $T_{B B N} \simeq 1 \mathrm{MeV}$, due to the sterile neutrinos is much less than that of an ordinary neutrino species. For sterile neutrinos that are much lighter than $1 \mathrm{MeV}$, this can be arranged simply by having them decouple early enough for their energy density to be diluted by the reheating of ordinary matter, such as in the QCD phase transition. But keeping in mind the supernova constraint of the previous section, the sterile neutrinos in the model of interest here satisfy $M \gg T_{B B N}$, and so even if they decouple sufficiently early, their relic energy density at nucleosynthesis will nevertheless dominate that of an ordinary neutrino species unless their lifetime is shorter than 0.1 sec. Assuming decays through the charged-current weak interactions, this implies:

$$
\left(\frac{M_{N_{ \pm}^{\prime}}}{\mathrm{GeV}}\right)^{5}\left|U_{e i}\right|^{2} \gtrsim 3.6 \times 10^{-11} .
$$

Suppose, first, that the sterile neutrino is relativistic at the time that it decouples: $T_{D} \gtrsim M_{N_{ \pm}^{\prime}}$. This is the case if

$$
\left(\frac{M_{N_{ \pm}^{\prime}}}{\mathrm{GeV}}\right)^{3}\left|U_{e i}\right|^{2} \lesssim 3 \times 10^{-8} .
$$

Both of these conditions must be satisfied by both of the heavy particles, and are plotted as curves $a$ and $b$ in Fig. (3). BBN demands that any particle which lies to the left of curve $b$ (i.e. decouples relativistically) must also lie to the right of curve $a$. Notice that these conditions together drive one, in the model of current interest, into a regime which is excluded by the laboratory bounds considered in the following section, provided one asks for a $\beta \beta_{0 \nu}$ rate close to observability at the same time, and so the relativistic-decoupling scenario is not relevant in our case.

Next suppose that the sterile neutrinos decouple nonrelativistically, $T_{D} \lesssim M_{N_{ \pm}^{\prime}}$, and so lie to the right of curve $b$ in Fig. (3). In this case their number density is exponentially 
suppressed by the Boltzmann factor. This dilution then makes them decouple earlier than one would otherwise expect without the Boltzmann suppression. The decoupling temperature, $T_{D}$, is therefore given by

$$
\frac{M_{N_{ \pm}}^{2} G_{F}^{2}\left|U_{e i}\right|^{2}}{\pi}\left(M_{N_{ \pm}} T_{D}\right)^{3 / 2} e^{-M_{N_{ \pm}} / T_{D}} \lesssim g_{\star}^{1 / 2} \frac{T_{D}^{2}}{M_{p l}}
$$

In the case of present interest the sterile neutrinos will always decouple at or above a temperature of $\sim 100 \mathrm{MeV}$, i.e. lie to the right or below curve $c$ in Fig. (3), provided $\beta \beta_{0 \nu}$ occurs at or close to an observable level. This in turn means that their energy density at nucleosynthesis will only be sufficiently small if they, again, decay fast enough, eq. (22).

To summarize, having observable $\beta \beta_{0 \nu}$ in this model implies that the heavy sterile neutrinos decouple only after having become non relativistic, but still early enough that conflict with standard Big-Bang Nucleosynthesis can be avoided, provided they decay fast enough.

\section{3) Laboratory Limits}

A wide range of experiments constrain the properties of isosinglet heavy leptons [2], [3], [4], [5], [6], [7]. They can be classified into two main categories, according to whether the bound is obtained from precision measurements on the $Z$ resonance, or from experiments at lower energies.

There are two main types of low-energy experiments which limit the properties of sterile neutrinos that mix with the electron neutrino, such as for the model considered here. One type obtains its bound from the decay rate and the electron spectrum of the two-body decay of kaons and pions at rest. For example, for neutrinos in the mass range between 1 and $100 \mathrm{MeV}$, the measured $\Gamma(\pi \rightarrow e \nu) / \Gamma(\pi \rightarrow \mu \nu)$ rate provides a massdependent bound on $\left|U_{e i}\right|$. For a $1 \mathrm{MeV}$ neutrino the bound is $\left|U_{e i}\right|^{2}<10^{-3}$ at the $90 \%$ C.L., whereas the respective bounds for a $10 \mathrm{MeV}$ and a $50 \mathrm{MeV}$ neutrino are $10^{-5}$ and $5 \times 10^{-7}[20]$. Similar searches for a nonstandard component to $K \rightarrow e \nu$ [21] extend this limit up to sterile-neutrino masses of $350 \mathrm{MeV}$.

Even stronger limits can be obtained from beam-dump experiments provided that the heavy neutral leptons can decay appreciably through their charged-current interactions. 
These can constrain sterile-neutrino masses up to $\sim 2 \mathrm{GeV}$, with a sensitivity of $\left|U_{e i}\right|^{2} \lesssim$ $10^{-7}$. For masses below $\sim 0.5 \mathrm{GeV}$ the bound becomes as good as $\left|U_{e i}\right|^{2} \sim 0.5 \times 10^{-9}$ [22]. (For a more detailed discussion see [3]).

For neutrinos with masses that are more than a few $\mathrm{GeV}$, measurements at the $Z$ pole provide the strongest limits. For $M_{N_{ \pm}^{\prime}}<M_{Z}$, the best bounds come from the nonobservation of the decay of a $Z$ into a sterile and a standard neutrino, $Z \rightarrow N \bar{\nu} \rightarrow W^{*} e \bar{\nu}$, with the subsequent decay of the sterile neutrino through a virtual boson, $W^{*}$. The bound obtained in this way is $\left|U_{e i}\right|^{2}<7 \times 10^{-5}$ [2], [4], [7].

The above bounds do not apply if the heavy singlet neutrino is heavier than $M_{Z}$. In this case there are two types of bounds to consider, which arise due to the reduction of the couplings of the ordinary neutrinos to the $W$ and $Z$ bosons due to their admixture with the new sterile neutrinos. The reduction in the effective couplings to the $Z$, result in a reduction of the $Z$ 's invisible width. This leads to the bound: $\left|U_{e i}\right|^{2}<2.7 \times 10^{-2}$ for isosinglet masses above $90 \mathrm{GeV}$ [6], [7]. A stronger bound arises however from the reduction of the $W$ couplings, which potentially show up as a failure of lepton universality in low-energy weak decays, as well as affecting precision electroweak measurements through their influence on the experimental value of Fermi's constant, $G_{F}$, that is inferred from muon decay. This implies the bound : $\left|U_{e i}\right|^{2}<5.6 \times 10^{-3}(2 \sigma)$ for isosinglet masses above $90 \mathrm{GeV}[6]$.

All the phenomenological constraints discussed in this section have been summarized in Fig. (3). When contrasted with the masses and mixing angles required for a $\beta \beta_{0 \nu}$ signal close to observability in the model discussed here (Figs. (1)) they yield the region of parameter space depicted in Fig. (4).

\section{Conclusions}

Our purpose here has been to determine which kinds of heavy sterile neutrinos can contribute appreciably to $\beta \beta_{0 \nu}$ decays, and to explore the constraints which such particles must satisfy due to present laboratory and astrophysical information. Part of our motivation for doing so has been to provide an example of a theory in which this decay can proceed without requiring the existence of a light neutrino with a mass in the vicinity of $1 \mathrm{eV}$. As an existence proof for theories of this type, we display here a model which does so. It does so by producing observable $\beta \beta_{0 \nu}$ purely through the exchange of a sterile neu- 
trino having a mass in the GeV range. This runs contrary to the usual expectation that the observation of $\beta \beta_{0 \nu}$ must indicate the existence of a majorana mass for the electron neutrino in the $e V$ range.

The model we consider is reasonably simple, supplementing the standard model only by two new left-handed neutrino states. We find that requiring the model to be consistent with all astrophysical and laboratory limits, as well as with an observable $\beta \beta_{0 \nu}$ signal, constrains the couplings and masses of the new neutrinos to lie in a limited region of parameter space. The mass range that is favoured by these bounds, as well as naturalness considerations, is $1-10 \mathrm{GeV}$. For these masses, the couplings that are required to produce an observable $\beta \beta_{0 \nu}$ signal are roughly $\left|U_{e i}\right|^{2} \sim 10^{-5}$. Such parameters place such a sterile neutrino close to the current limits of detection at LEP, where they can be searched for

through the decay $Z \rightarrow N \nu_{e}$, with the subsequent charged-current decay of the sterile neutrino, $N$, into quarks and leptons. This shows how LEP results can be used to help diagnose the implications of a potential $\beta \beta_{0 \nu}$ signal, and illustrates the rich interplay that is possible between low- and high-energy experiments.

\section{Acknowledgments}

We would like to acknowledge helpful conversations with K. Babu, as well as research support from N.S.E.R.C. of Canada, les Fonds F.C.A.R. du Québec, the U.S. National Science Foundation (grant PHY-9119745) and the Swiss National Foundation. 


\section{References}

[1] M. Gell-Mann, P. Ramond and R. Slansky, in Supergravity, ed. by F. van Nieuwenhuizen and D. Freedman (Amsterdam, North Holland, 1979) 315;

T. Yanagida, in the Proceedings of the Workshop on Unified Theory and Baryon Number in the Universe, ed. by O. Sawada and A. Sugamoto (KEK, Tsukuba, 1979) 95 ;

R.N. Mohapatra and G. Senjanović, Phys. Rev. Lett. 44 (1980) 912.

[2] The L3 Collaboration, Phys. Lett. 316B (1993) 427; S. Shevchenko, preprint CALT68-1939 (1993).

[3] M. Gonau, C. Leung and J. Rosner, Phys. Rev. D29 (1984) 2539; F.J. Gilman, Comments Nucl. Part. Phys. 16 (1986) 231.

[4] M. Dittmar, M.C. Gonzalez-Garcia, A. Santamaria and J.W.F. Valle, Nucl. Phys. B332 (1990) 1.

[5] A. Pilaftsis, Phys. Rev. D49 (1993) 2398.

[6] C.P. Burgess, S. Godfrey, H. König, D. London and I. Maksymyk, Phys. Rev. D49 (1994) 6115.

[7] L.N. Chang, D. Ng and J. Ng, preprint TRI-PP-94-1.

[8] R. N. Mohapatra, Phys. Rev. D34 (1986) 3457; R. N. Mohapatra and J. D. Vergados, Phys. Rev. Lett. 47 (1981) 1713; J. Schecter and J. W. F. Valle, Phys. Rev. D25 (1982) 2951.

[9] C.N. Leung and S.T. Petcov, Phys. Lett. 145B (1984) 416.

[10] For an excellent discussion of Majorana neutrinos and their connection to $\beta \beta_{0 \nu}$ decay,see B. Kayser, F. Gibra-Debu and F. Perrier, Physics of Massive Neutrinos, ( World Scientific, 1989).

[11] C.P. Burgess and J.M. Cline, Phys. Lett. 289B (1993) 141; Phys. Rev. D49 (1994) 
5925.

[12] A. Halprin, P. Minkowski, H. Primakoff and P. Rosen, Phys. Rev. D13 (1976) 2567.

[13] W. Haxton and G. Stephenson, Prog. in Particle and Nucl. Physics 12, 409 (1984); J. Vergados, Phys. Rep. 133, 1 (1986); M. Doi, T. Kotani, H. Nishiura and E. Takasugi, Prog. Theor. Phys., Prog. Theor. Phys. Suppl. 83, 1 (1985).

[14] H. Klapdor-Kleingrothaus et.al., Heidelberg Preprint, (1993).

[15] A. Piepke, TAUP '93, ( to appear in the proceedings); Older references are: A. Piepke, presented at ICHEP XXVI, Dallas, Aug. 1992; D. Caldwell et.al., Nucl. Phys. (Proc. Suppl.) B13 1990 547; Int. J. Mod. Phys. A4 (1989) 1851; M. Moe and P. Vogel, Ann. Rev. of Nucl. and Part. Sc. (to appear).

[16] T. Tomoda, Rep. Prog. Phys. 54 (1991) 53.

[17] R. Barbieri and R.N. Mohapatra, Phys. Rev. D39 (1989) 1229; G. Raffelt and D. Seckel, Phys. Rev. Lett. 60 (1989) 1793.

[18] See the NEMO III Proposal, (1994).

[19] T. Walker et.al., Ap. J. 376, 51(1991).

[20] D.I. Britton et.al., Phys. Rev. D46 (1992) R885; Phys. Rev. Lett. 68 (1992) 3000.

[21] R. Shrock, Phys. Lett. 96B (1980) 159; T. Yamazaki et.al., in Proc. XIth Intern. Conf. on Neutrino Physics and Astrophysics, K. Kleinknecht and E.A. Paschos (World Scientific, Singapore, 1984) p. 183.

[22] G. Bernardi et.al., Phys. Lett. 203B (1988) 332; J. Dorenbosch et.al., Phys. Lett. 166B (1986) 473; R.C. Ball et.al., preprint UM HE 85-09, (1985) unpublished; A.M. Cooper-Sarkar et.al., Phys. Lett. 160B (1985) 207. 


\section{Figure Captions}

(1) This figure plots the region in the $U_{e i}-m_{i}$ plane (Fig. 1a, (1b) for $N_{+}^{\prime},\left(N_{-}^{\prime}\right.$ respectively)) that is allowed by current $\beta \beta_{0 \nu}$ experiments for various different values of the parameter $\epsilon$ as defined in eq. (8). The area below the corresponding lines is the allowed region. The limiting case of very large $\epsilon$ appears as the solid line labelled " $\epsilon=\infty$ ", which also corresponds to the contribution of a single sterile neutrino that mixes with $\nu_{e}$.

(2) The Feynman graph which dominates the renormalization-group mixing of $\mu_{-}$with $\tilde{\lambda}_{e}$ and $\mu_{+}$.

(3) This figure summarizes the phenomenological constraints as discussed in section 4 . The region above the solid line is ruled out by the various laboratory bounds. The region below and to the left of the dashed line labelled $S N$ is excluded by the observations of SN1987a. The dashed line labelled $N N$ depicts the naturalness bound as discussed in section 3 (Eq. (20)) for $\epsilon=0.1$. In the region to the right and above this line fine tuning is required. Finally the dash-dotted curves labelled $a, b$ and $c$ represent the nucleosynthesis bounds. The lifetime of a sterile neutrino is less than 0.1 sec to the right of curve $a$. A particle decouples after having become non relativistic in the region to the right of line $b$, but it will nevertheless decouple at or above a temperature of $\sim 100 \mathrm{MeV}$ to the right and below line $c$. Line $a$ thus represents the only relevant nucleosynthesis bound in our case, and the region to the right of line $a$ and below the solid line is allowed.

(4) We plot here the region of the parameter space in the $\epsilon-M_{N_{+}^{\prime}}$ plane which is obtained by requiring masses and mixing angles which yield a $\beta \beta_{0 \nu}$ signal close to observability, as depicted by the various lines in Fig. (1), together with the various phenomenological bounds displayed in Fig. (3). The allowed area is marked by shading, and extends upwards beyond the region depicted in the figure towards higher values of $\epsilon$ without changing the mass range. The darker area represents the part of the parameter space in which the smallness of the $\nu_{e}^{\prime}$ mass is explained in a way which is technically natural in the sense explained in section (3.1) of the text. In the lightly-shaded region a finetuning of $\tilde{\lambda}_{e}$ and $\mu_{+}$is required in order to keep the $\nu_{e}^{\prime}$ mass below $1 \mathrm{eV}$. Notice 
that, since $M_{N_{-}^{\prime}} \geq M_{N_{+}^{\prime}}$, the analogous figure in the $\epsilon-M_{N_{-}^{\prime}}$ plane would be shifted to the right according to eq. (9). 
This figure "fig1-1.png" is available in "png" format from: http://arxiv.org/ps/hep-ph/9408367v2 

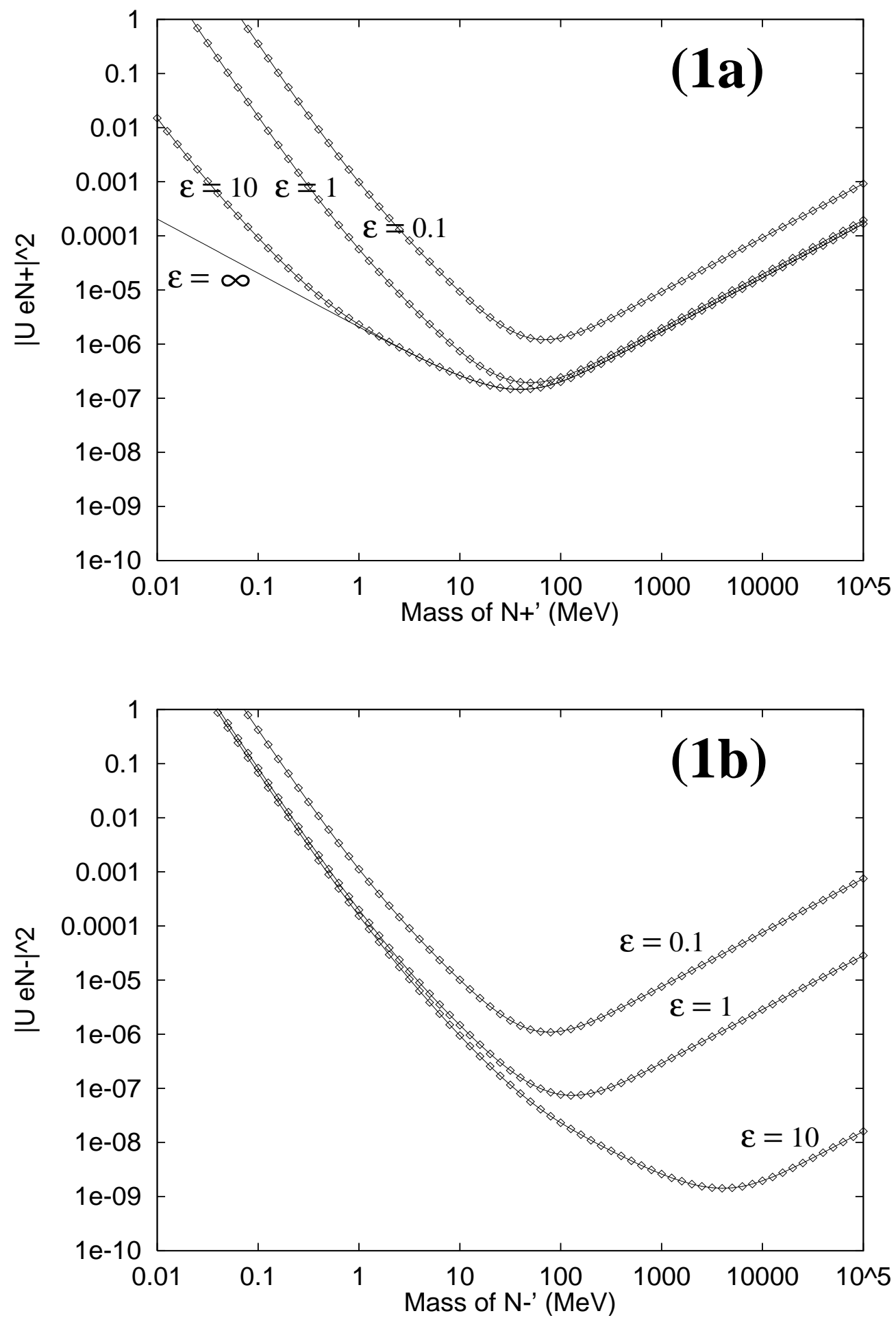
This figure "fig1-2.png" is available in "png" format from: http://arxiv.org/ps/hep-ph/9408367v2 
(2)

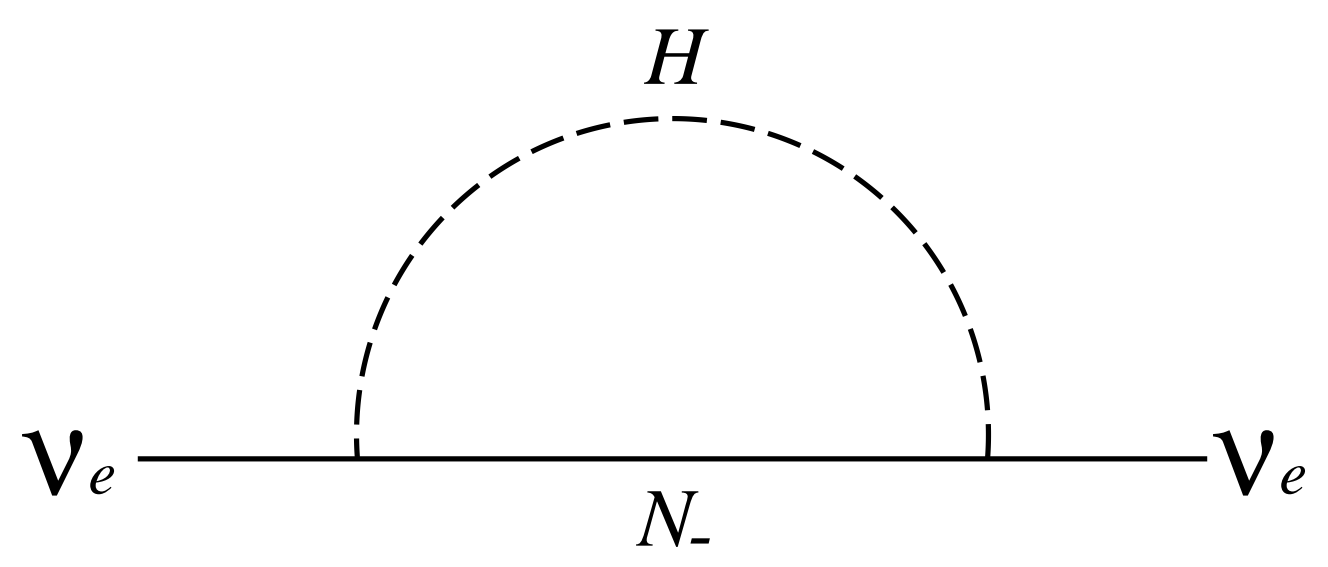


This figure "fig1-3.png" is available in "png" format from: http://arxiv.org/ps/hep-ph/9408367v2 


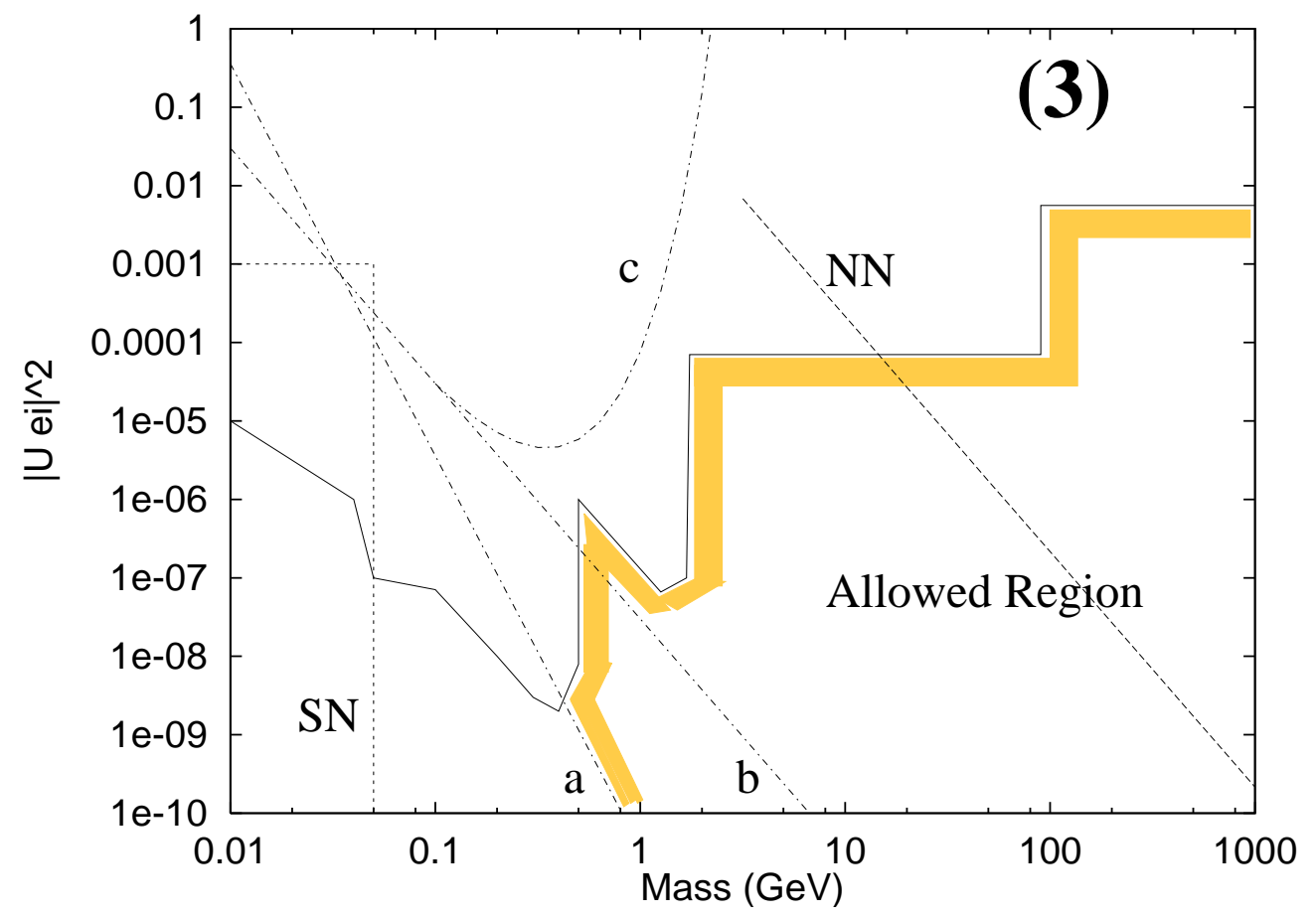


This figure "fig1-4.png" is available in "png" format from: http://arxiv.org/ps/hep-ph/9408367v2 


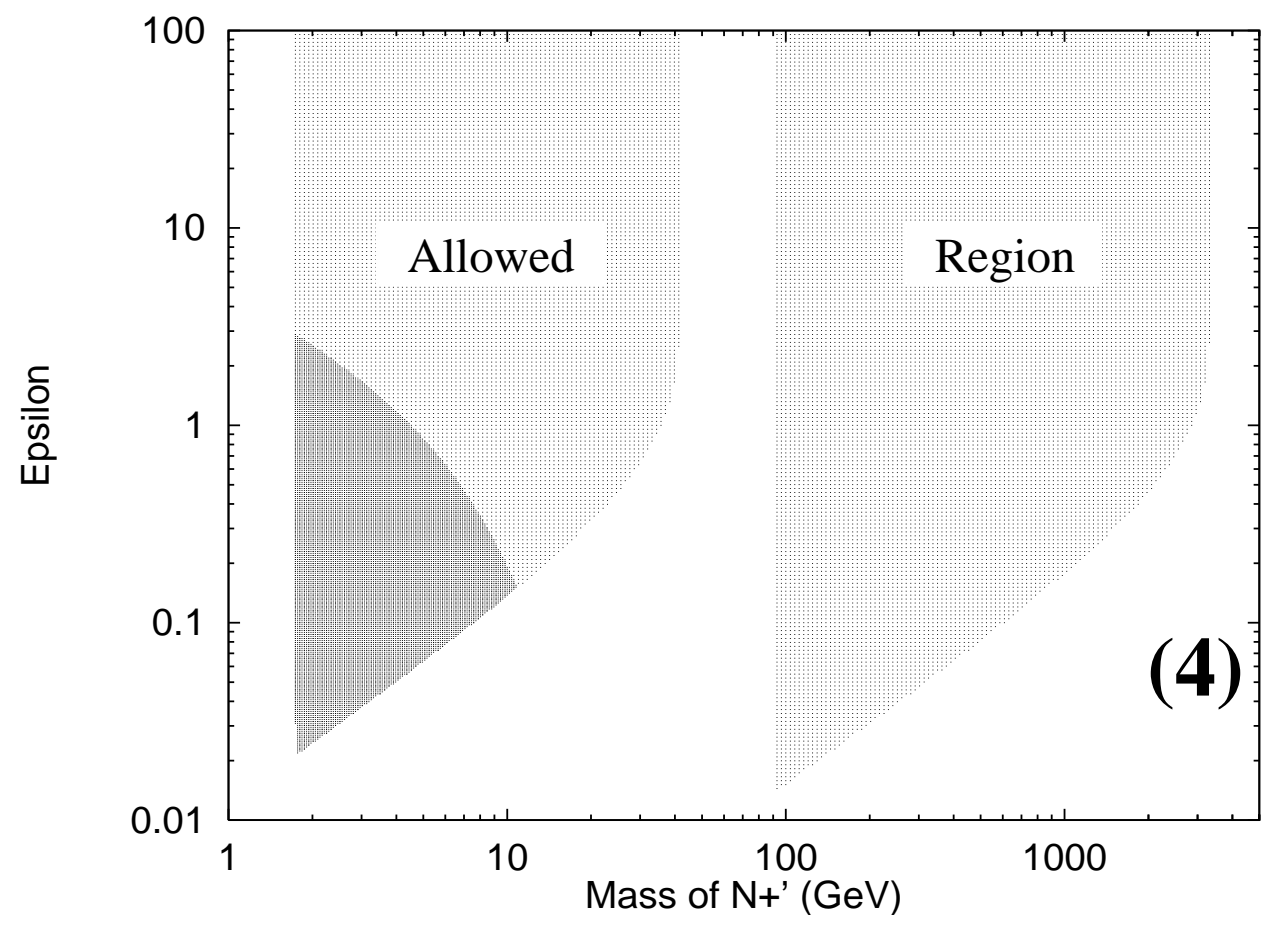

